and Professor R. J. Elliott and Dr. Richard Forman for several discussions on the properties of cuprous oxide. We also wish to thank Dr. Forman, W. S. Brower, Jr., and H. S. Parker for the generous loan of the crystal used in our experiments.

†Work supported by the National Science Foundation.

*Alfred P. Sloan Research Fellow.

${ }^{1}$ E. F. Gross, Usp. Fiz. Nauk 76, 433 (1962), and 103, 431 (1971) [Sov. Phys. Usp. $\underline{5}, \overline{195}(1962)$ and $\underline{14}, 104$ (1971)]

${ }^{2}$ A. Compaan and H. Z. Cummins, Phys. Rev. B $\underline{6}$, 4753 (1972).

${ }^{3}$ Y. Petroff, P. Y. Yu, and Y. R. Shen, Phys. Rev. Lett. 29,1558 (1972).

${ }^{4}$ P. Y. Yu, Y. R. Shen, Y. Petroff, and L. M. Falicov, Phys. Rev. Lett. 30, 283 (1973).

${ }^{5}$ E. F. Gross, A. G. Zhilich, B. P. Zakharchenya, and A. V. Varfalomeev, Fiz. Tverd. Tela $\underline{3}$, 1445 (1961) [Sov. Phys. Solid State $\underline{3}, 1048$ (1961)].

${ }^{6}$ S. Nikitine, J. B. Grun, and M. Certier, Phys. Kondens. Mater. 1,214 (1963).
${ }^{7}$ W. S. Brower, Jr., and H. S. Parker, J. Cryst. Growth 8,227 (1971).

${ }^{8}$ A. Compaan and H. Z. Cummins, Bull. Amer. Phys. Soc. 18, 75 (1973).

${ }^{9}$ Air Products and Chemicals, Inc., Allentown, Pa.

${ }^{10}$ The data points in Fig. 2 indicate the observed Raman intensity divided by the incident laser power. Absorption corrections, which are less than $4 \%$ in this frequency range, have not been included.

${ }^{11}$ R. Loudon, Advan. Phys. 13, 423 (1964); J. L. Birman, "Theory of Crystal Space Groups and Infra-Red and Raman Processes in Insulating Crystals" in Handbuch der Physik, edited by S. Flügge (Springer, New York, 1973, to be published), Vol. 25, Part 2b.

${ }^{12}$ See, for example, M. Born and M. Bradburn, Proc. Roy. Soc., Ser. A 188, 161 (1947); L. N. Ovander, Opt. Spectrosk. 9, 571 (1960) [Opt. Spectrosc. 9 , 302 (1960)]. ${ }^{13} \mathrm{~J}$. L. Birman, to be published.

${ }^{14}$ P. F. Williams and S. P. S. Porto, Bull. Amer. Phys. Soc. 18, 411 (1973); P. F. Williams, Ph. D. thesis, University of Southern California, 1973 (unpublished).

${ }^{15}$ P. Y. Yu, Y. R. Shen, and Y. Petroff, Bull. Amer. Phys. Soc. 18, 411 (1973).

${ }^{16}$ R. M. Martin, Phys. Rev. B $\underline{4}, 3676$ (1971).

\title{
Hopping Conductivity in Granular Metals
}

\author{
Ping Sheng \\ Institute for Advanced Study, Princeton, New Jersey 08540
}

and

B. Abeles and Y. Arie

$R C A$ Laboratories, Princeton, New Jersey 08540

(Received 23 April 1973)

\begin{abstract}
We present evidence that in granular metals the observed temperature dependence of the low-field conductivity, $\exp \left(-b / T^{\alpha}\right)$ with $\alpha=\frac{1}{2}$, can be ascribed to a relationship $s E_{c}$ $=$ const between $s$, the separation of neighboring metal grains, and $E_{c}$, the electrostatic energy required to create a positive-negative charged pair of grains. This relationship results from simple considerations of the structure of granular metals. The predictions of the theory, for both the high- and the low-field electrical conductivity, are in excellent accord with experimental results in granular $\mathrm{Ni-SiO}{ }_{2}$ films.
\end{abstract}

The low-field electrical conductivity $\sigma_{L}$ of many disordered materials has a temperature dependence that can be expressed in the form $\sigma_{L} \sim \exp \left(-b / T^{\alpha}\right)$. The value $\alpha=\frac{1}{4}$, found in many of the amorphous semiconductors and semiconducting glasses, has been predicted by Mott ${ }^{1}$ using a model of hopping conductivity between localized states. There are, however, disordered materials such as granular metals,,$^{2-4}$ and some disordered semiconductors, ${ }^{5}$ which exhibit an $\alpha=\frac{1}{2}$ behavior for which no definitive theory has been proposed. In this Letter we present evi- dence that in granular metals, consisting of fine metallic particles dispersed in a dielectric matrix, the $\alpha=\frac{1}{2}$ behavior can be explained by a structural effect. Furthermore, the theory we propose predicts the temperature and electric field dependences of conductivity in the high-field regime and relates the high-and low-field behaviors through a single structural parameter. We present measurements of the temperature and field dependences of the resistivities in granular $\mathrm{Ni}-\mathrm{SiO}_{2}$ films and find excellent agreement with the predictions of the theory. 


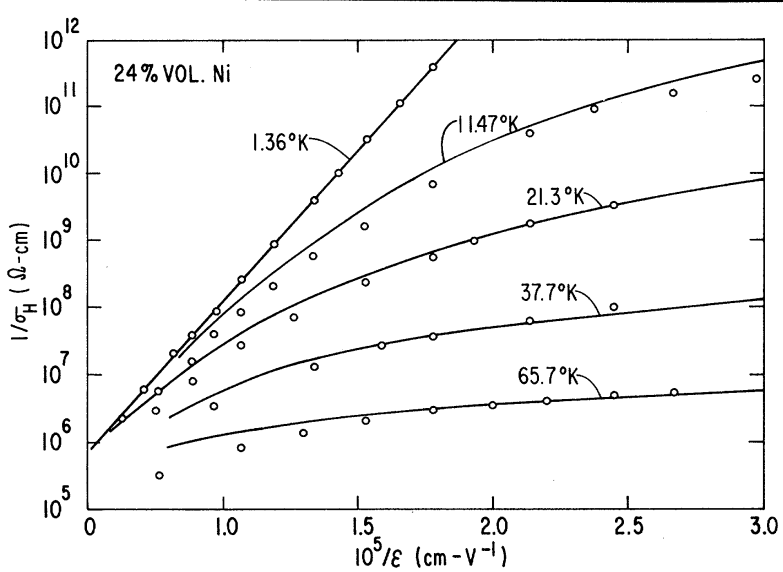

FIG. 1. The high-field resistivity $1 / \sigma_{H}$ as a function of the reciprocal electric field $1 / \mathscr{E}$ for several temperatures of a $\mathrm{Ni}-\mathrm{SiO}_{2}$ film, measured with the electric field normal to the film. The composition is indicated in the figure. The solid lines were calculated from Eq. (2) with the values of the parameters $\mathcal{E}_{0}$ and $2\left(C_{0} / k\right)^{1 / 2}$ given in columns 2 and 3 of Table I.

The $\mathrm{Ni}-\mathrm{SiO}_{2}$ films, about $1000 \AA$ thick, were prepared $^{6.7}$ by cosputtering $\mathrm{Ni}$ and silica. Electron microscopy ${ }^{8,9}$ and $\mathrm{x}$-ray analysis ${ }^{9}$ show that the films consist of small crystalline nickel grains embedded in amorphous $\mathrm{SiO}_{2}$. The resistivities, high-field and low-field, are presented in Figs. 1 and 2.

Electrical conduction in granular metals results from the transport of electrons and holes by tunneling from charged metal grains to neutral grains. ${ }^{10}$ In order to generate a charge carrier, an electron has to be removed from a neutral grain and placed on a neighboring neutral grain. Such a process requires an energy $E_{c}$ $=\left(e^{2} / d\right) F(s / d)$, where $e$ is the electronic charge, $d$ is the grain size, $s$ is the separation between the grains, and $F$ is a function whose form depends on shape and arrangement of the grains. ${ }^{11}$ In the low-field regime the charge carriers are thermally activated. In the high-field regime where the voltage drop, $\Delta V$, between neighboring grains is much larger than $k T / e$, where $k$ is the Boltzmann constant, the majority of charge carriers are created by field-induced tunneling between neutral grains. ${ }^{7}$ From the above discussion, it is clear that the charging energy $E_{c}$ plays a central role in the granular-metal conduction process, and, therefore, a closer look at the structural origin of $E_{c}$ is necessary. We now propose, using a simple plausibility argument, a structural relationship between $E_{c}$ and $s$. It is known from electron micrographs that

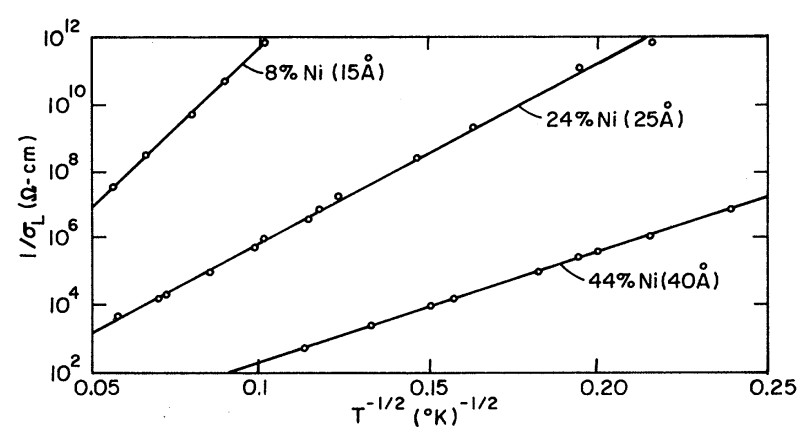

FIG. 2. The low-field resistivity $1 / \sigma_{L}$ as a function of $T^{-1 / 2}$ for three different compositions of $\mathrm{Ni}-\mathrm{SiO}_{2}$ films. The compositions and the corresponding mean sizes of the nickel grains are indicated in the figure. The full lines represent the relation $\log \sigma_{L}=-2\left(C_{0} /\right.$ $k T)^{1 / 2}$; the values of $2\left(C_{0} / k\right)^{1 / 2}$ are given in column 4 of Table I.

in granular metals there is a distribution of grain sizes. Since the grains are formed by surface diffusion of the sputtered nickel atoms and $\mathrm{SiO}_{2}$ molecules, the composition averaged over a volume larger than the surface diffusion length, which is of the order of a few grains, is expected to be the same throughout the volume of the sample and equal to that of the bulk. The condition of constant composition requires $s$ and $d$ to be related. Consider the granular metal to be divided into regions each of which contains grains of roughly the same size. In order for the composition of each of the se regions to be equal to the bulk composition, it is necessary for the ratio $s / d$ to have the same value for each region even though $d$ is allowed to vary from region to region. It follows that $s E_{c}$ is a constant whose value depends only on the composition of the sample and the dielectric constant of the insulator.

We now derive, on the basis of the rule $s E_{c}$ = const, the high-field and the low-field behaviors of the conductivity in granular metals. ${ }^{12}$ For the purpose of calculation we use for the distribution function $D(s)$ of $s$ the form $D(s)=(s /$ $\left.s_{0}{ }^{2}\right) \exp \left(-s / s_{0}\right)$, where $s_{0}$ is the most probable value of $s$. This form of distribution function is an approximation to the one determined from electron micrographs.

(a) High-field regime. - In this limit the current density $j$ is proportional to the generation rate of charge carriers through field-induced tunneling. ${ }^{70} 9$ To simplify the calculation we assume that the applied electric field $\mathcal{E}$ gives rise to equal voltage drops $\Delta V=\mathcal{E} \Delta l$ between layers of neighboring grains. The layers are separated by $\Delta l$, and on the average are perpendicular to 
the direction of the macroscopic field. The rate of tunneling for an electron with energy $E$ from a grain on one layer to a neighboring grain on the next layer is $\sim \exp (-2 \chi s) f(E)\left[1-f\left(E+e \Delta V-E_{c}\right)\right]$, where $f(E)=1 /[1+\exp (E / k T)]$ is the Fermi function, and $\chi=\left[2 m(\varphi-E) / \hbar^{2}\right]^{1 / 2}$ with $\varphi$ the effective barrier height. The current density $j$ can be written as

$$
j=\text { const } \int_{0}^{\infty} d s D(s) \int_{-\infty}^{\infty} e^{-2 x s} f(E)\left[1-f\left(E+e \Delta V-E_{c}\right)\right] d E .
$$

The current term due to electrons tunneling counter to the applied electric field, which is negligible for the case $e \Delta V \gg k T$, has been dropped in Eq. (1). Since in our system the barrier height (3.6 eV) is significantly larger than $e \Delta V$, we have neglected in Eq. (1) the effect of applied field on the barrier shape, and have regarded $\chi$ as a constant $\simeq\left(2 m \varphi / \hbar^{2}\right)^{1 / 2}$. A change of variables in Eq. (1) and integration with respect to $E$ yields

$$
j / \mathscr{E}=\sigma_{H}=\sigma_{0} \exp \left(-\mathcal{E}_{0} / \mathscr{E}\right) \int_{-\mathcal{E}_{0} / \mathcal{E}}^{\infty} \exp (-Z) Z d Z\left\{1-\exp \left[-\frac{Z}{Z+\left(\mathcal{E}_{0} / \mathcal{E}\right)} \frac{C_{0} \mathscr{E}}{\mathcal{E}_{0} k T}\right]\right\}^{-1} .
$$

Here $C_{0}=2 \chi s E_{c}\left[1+1 /\left(2 \chi s_{0}\right)\right]$, and $\mathcal{E}_{0}=C_{0} / \Delta l e$. At $T=0 \mathrm{Eq}$. (2) reduces to $\sigma=\sigma_{0} \exp \left(-\mathcal{E}_{0} / \mathscr{E}\right)$. This behavior can be qualitatively understood as follows. When the voltage difference between the neighboring layers of grains is $\Delta V$, the electron can only tunnel between those pairs of neighboring grains with $E_{c} \leqslant e \Delta V$. Since $s E_{c}=$ const, the rate of tunneling is $\sim \exp (-2 \chi s)=\exp$ (- const/ $\left.E_{c}\right) \cong \exp (-$ const $/ e \Delta V)$. With $\mathcal{E}_{0}$ determined by the zero-temperature behavior, Eq. (2) represents a one-parameter $\left(C_{0}\right)$ description of the temperature and field dependences of high-field conductivity. Figure 1 shows the experimental data and the theoretical curves calculated from Eq. (2) with $C_{0}$ as the fitting parameter. Because of lack of space only the data for one of the granular $\mathrm{Ni}-\mathrm{SiO}_{2}$ compositions are shown. The fits are equally good for other compositions.

(b) Low-field regime. - In this limit the charge carriers are thermally activated. We assume that the generated positive-negative charged pair of grains are roughly of same size so that each contributes about half of the charging energy $E_{c}$. Let us consider all those charge carriers with charging energy $E_{c}^{\prime}$. Their number density is $\sim \exp \left(-E_{c}{ }^{\prime} / k T\right)$. When a weak electric field $(e \Delta V \ll k T)$ is applied, the charge will drift along a path of largest mobility towards the electrodes. The charge is inhibited in tunneling to a much smaller grain with $E_{c} \gg E_{c}^{\prime}$ because the electron has insufficient energy. The charge will, therefore, tunnel to grains with $E_{c} \leqslant E_{c}{ }^{\prime}$. However, since smaller $E_{c}$ is associated with a larger tunneling barrier by the rule $s E_{c}=$ const, the optimal path follows the regions with the least deviations of $E_{c}$ from $E_{c}{ }^{\prime}$. The corresponding mobility is proportional to $\exp \left(-2 \chi s^{\prime}\right)$, where $s^{\prime}$ $=$ const $/ E_{c}{ }^{\prime}$, and the total conductivity, being the sum of products of mobility, charge, and number density of charge carriers over all charging energies, can be written as

$$
\sigma_{L} \sim \int_{0}^{\infty} d s^{\prime} D\left(s^{\prime}\right) \exp \left[-2 \chi s^{\prime}-\left(E_{c}^{\prime} / k T\right)\right]
$$

By performing the integration by the method of steepest descent, the dominant temperature dependence of the low-field conductivity is given by $\sigma_{L} \sim \exp \left[-2\left(C_{0} / k T\right)^{1 / 2}\right]$. The pre-exponential factor of the conductivity is not given by the above argument because its exact form depends on more complicated considerations of statistical fluctuations and the counting of percolation paths. However, the temperature dependence of the pre-exponential factor is expected to be weak.

In Table I we give the values of $\mathcal{E}_{0}$ and compare the values of $2\left(C_{0} / k\right)^{1 / 2}$ obtained from the slopes of $\ln \sigma_{L}$ versus $1 / \sqrt{T}$ plots (Fig. 2) with those obtained from the high-field data. The agreement between the two sets of values of $2\left(C_{0} / k\right)^{1 / 2}$ is truly remarkable in view of the fact that two different physical mechanisms, field generation in the high-field regime and temperature activation with percolation mobility in the low-field regime, are operative in the two limits. Direct calculations of $C_{0}$ from the expression $2 \chi s E_{c}\left(1+1 / 2 \chi s_{0}\right)$ were found to be in agreement with those in $\mathrm{Ta}-$ ble I within the uncertainties of the parameters, e.g., for the sample with $24 \%$ volume $\mathrm{Ni}$, if we

\begin{tabular}{|c|c|c|c|}
\hline \multirow[b]{2}{*}{$\%$ vol Ni } & \multirow{2}{*}{$\begin{array}{c}\mathcal{E}_{0} \\
\left(10^{6} \mathrm{~V} / \mathrm{cm}\right)\end{array}$} & \multicolumn{2}{|c|}{$\begin{array}{c}2\left(C_{0} / k\right)^{1 / 2} \\
\left({ }^{0} \mathrm{~K}^{1 / 2}\right)\end{array}$} \\
\hline & & $\mathcal{E}$ dependence & $T$ dependence \\
\hline 8 & 3.50 & 160 & 230 \\
\hline 24 & 1.04 & 106 & 122 \\
\hline 44 & 0.23 & 56 & 69 \\
\hline
\end{tabular}
take $\chi=1 \AA^{-1}$ (Ref. 7), $s=5 \AA$ (estimated from

TABLE I. Parameters of the granular metals. 
electron microscopy), and $E_{c}=30 \mathrm{meV}$ (estimated from a plot of $\ln \sigma_{L}$ versus $1 / T$ ), we obtain $2\left[\left(C_{0} / k\right)^{1 / 2}\right] \approx 120\left({ }^{\circ} \mathrm{K}\right)^{1 / 2}$. The self-consistency of the model receives further confirmation in that the separation between the layers of grains calculated from the relation $\mathscr{E}_{0}=C_{0} / \Delta l e$ agrees with the mean value of $s+d$ determined from the electron microscopy.

It is instructive to point out the similarities and the differences between Mott's model and ours. In Mott's model the density of charge carriers is assumed to be temperature independent, and the percolation paths for the charge carriers are determined by optimizing the mobility. In our model the charge carriers are thermally activated, tunneling occurs between nearest neighbors only, and the optimization is applied to the product of mobility and number density of charge carriers. The differences between charging energies in our model are analogous to the relative displacements of the energy levels for the localized states in Mott's model. However, in our case not only the differences but also the magnitudes of $E_{c}$ play an important role. This is especially obvious in the high-field regime where the governing factor for field generation of charge carriers is the value of $E_{c}$ rather than the differences in $E_{c}$.

To conclude, we would like to make the following comments: The field dependence, $\exp \left(-\mathcal{E}_{0} /\right.$ $\mathcal{E})$, of $\sigma_{H}$ in the low-temperature regime is partially the result of high tunneling barriers in $\mathrm{Ni}-\mathrm{SiO}_{2}$. In materials where the tunneling barrier is low, the high-field conductivity might well follow some other form of behavior (such as the Frenkel-Poole effect). However, the relationship $s E_{c}=$ const should still yield the $\alpha=\frac{1}{2}$ behavior in the low-field regime. Attemps are being made to extend the concept of structural effects to other disordered materials. It is interesting to note that the inhomogeneous transport regime in disordered materials, recently treated by Cohen and Jortner, ${ }^{13}$ bears close analogy to granular metals.

${ }^{1}$ N. F. Mott, Phil. Mag. 19, 835 (1969); V. Ambegaokar, B. I. Halperin, and J.S. Langer, Phys. Rev. B $\underline{4}$, 2612 (1971).

${ }^{2}$ J. I. Gittleman, Y. Goldstein, and S. Bozowski, Phys. Rev. B 5, 3609 (1972).

${ }^{3}$ H. R. Zeller, Phys. Rev. Lett. 28, 1452 (1972).

${ }^{4}$ J. J. Hauser, Phys. Rev. B (to be published).

${ }^{5}$ D. Redfield, Bull. Amer. Phys. Soc. 18, 361 (1973), and Phys. Rev. Lett. 30, 1319 (1973).

${ }^{6}$ J. J. Hanak, H. W. Lehmann, and R. K. Wehner, J. Appl. Phys. 43, 1666 (1972).

${ }^{7}$ P. Sheng and B. Abeles, Phys. Rev. Lett. 28, 34 (1972).

${ }^{8}$ M. S. Abrahams, C. J. Buiocchi, M. Rayl, and P. J. Wojtowicz, J. Appl. Phys. 43, 2537 (1972).

${ }^{9} \mathrm{~B}$. Abeles, P. Sheng, M. Coutts, and Y. Arie, to be published, will contain more experimental and theoretical details.

${ }^{10} \mathrm{C}$. A. Neugebauer and M. B. Webb, J. Appl. Phys. $\underline{33}$, 74 (1962); C. A. Neugebauer, Thin Solid Films $\underline{6}, 44 \overline{3}$ (1970).

${ }^{11}$ In Ref. 7, for example, $F(s / d)=2(s / d) / \kappa\left[\frac{1}{2}+(s / d)\right]$, where $\kappa$ is the dielectric constant.

${ }^{12}$ The present treatment of high-field conductivity differs from that in Ref. 7 in that we no longer consider $E_{c}$ as constant.

${ }^{13} \mathrm{M}$. H. Cohen and J. Jortner, Phys. Rev. Lett. 30 , 699 (1973); R. Landauer, J. Appl. Phys, 23 , 779 (1952).

\title{
Scaling Theory for Metastable States and Their Lifetimes
}

\author{
K. Binder* and E. Stoll \\ IBM Zurich Research Laboratory, 8803 Rüschlikon, Switzerland
}

(Received 6 April 1973)

\begin{abstract}
The response of the magnetization to a sudden reversal of the magnetic field is studied in the kinetic Ising model by means of computer experiments on square $N \times N$ lattices. It is found that the nonequilibrium relaxation function fulfills a dynamic scaling hypothesis. The magnetization of the metastable state agrees with predictions of the cluster model and also with an analytic continuation of the linear-model equation of state.
\end{abstract}

Apart from systems where the mean-field approximation is valid, ${ }^{1}$ no reliable predictions about the properties of metastable states exist. Monte Carlo calculations have been performed on the $N \times N$ square kinetic Ising model, ${ }^{2,3}$ and we obtained the magnetization of the metastable states, their lifetimes, and the detailed nonequilibrium behavior. ${ }^{3}$ Within the accuracy of the numerical calculations (roughly 1\%) the results agree with simple scaling ideas. 